\title{
Epigallocatechin-3-Gallate Promotes Osteo-/Odontogenic Differentiation of Stem Cells from the Apical Papilla through Activating the BMP-Smad Signaling Pathway
}

\author{
Zeni Liu, Yuxiu Lin, Xiaolin Fang, Jingwen Yang * and Zhi Chen *(D)
}

Citation: Liu, Z.; Lin, Y.; Fang, X.; Yang, J.; Chen, Z.

Epigallocatechin-3-Gallate Promotes Osteo-/Odontogenic Differentiation of Stem Cells from the Apical Papilla through Activating the BMP-Smad Signaling Pathway. Molecules 2021, 26 , 1580. https://doi.org/10.3390/ molecules 26061580

Academic Editors: Zohaib Khurshid, Paulo J. Palma, Farshid Sefat and Jason Peter Mansell

Received: 5 February 2021

Accepted: 9 March 2021

Published: 12 March 2021

Publisher's Note: MDPI stays neutral with regard to jurisdictional claims in published maps and institutional affiliations.

Copyright: (c) 2021 by the authors. Licensee MDPI, Basel, Switzerland. This article is an open access article distributed under the terms and conditions of the Creative Commons Attribution (CC BY) license (https:// creativecommons.org/licenses/by/ $4.0 /)$.
The State Key Laboratory Breeding Base of Basic Science of Stomatology (Hubei-MOST) \& Key Laboratory of Oral Biomedicine Ministry of Education, School and Hospital of Stomatology, Wuhan University,

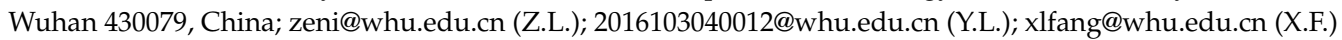

* Correspondence: jingwen.yang@whu.edu.cn (J.Y.); zhichen@whu.edu.cn (Z.C.)

Abstract: Stem cells from apical papilla (SCAPs) are desirable sources of dentin regeneration Epigallocatechin-3-gallate (EGCG), a natural component of green tea, shows potential in promoting the osteogenic differentiation of bone mesenchymal stem cells. However, whether EGCG regulates the odontogenic differentiation of SCAPs and how this occurs remain unknown. SCAPs from immature human third molars (16-20 years, $n=5)$ were treated with a medium containing different concentrations of EGCG or bone morphogenic protein 2 (BMP2), with or without LDN193189 (an inhibitor of the canonical BMP pathway). Cell proliferation and migration were analyzed using a CCK-8 assay and wound-healing assay, respectively. Osteo-/odontogenic differentiation was evaluated via alkaline phosphatase staining, alizarin red S staining, and the expression of osteo/odontogenic markers using qPCR and Western blotting. We found that EGCG (1 or $10 \mu \mathrm{M})$ promoted the proliferation of SCAPs, increased alkaline phosphatase activity and mineral deposition, and upregulated the expression of osteo-/odontogenic markers including dentin sialophosphoprotein (Dspp), dentin matrix protein-1 (Dmp-1), bone sialoprotein (Bsp), and Type I collagen (Col1), along with the elevated expression of BMP2 and phosphorylation level of Smad1/5/9 $(p<0.01)$. EGCG at concentrations below $10 \mu \mathrm{M}$ had no significant influence on cell migration. Moreover, EGCG-induced osteo-/odontogenic differentiation was significantly attenuated via LDN193189 treatment $(p<0.01)$. Furthermore, EGCG showed the ability to promote mineralization comparable with that of recombinant BMP2. Our study demonstrated that EGCG promotes the osteo-/odontogenic differentiation of SCAPs through the BMP-Smad signaling pathway.

Keywords: stem cells from apical papilla; epigallocatechin-3-gallate; osteo-/odontogenic differentiation; proliferation; BMP-Smad signaling pathway

\section{Introduction}

Irreversible pulpitis and pulp necrosis in developing teeth usually lead to the formation of nonvital pulp tissue and the ceasing of root development. The emergence of regenerative endodontics provides a promising approach to treating these diseases [1-3]. Stem cells from apical papilla (SCAPs) are a population of cells existing in the root apex of the immature tooth, contributing to root dentin formation and root elongation during root maturation [4,5]. SCAPs exhibit great viability and potency of multipotent differentiation, including odontogenic, osteogenic, neurogenic, and adipogenic differentiation [6-8]. Furthermore, SCAPs, along with periodontal ligament stem cells (PDLSCs), can regenerate a bio-root with periodontal ligament in vivo [6]. Therefore, SCAPs are considered to be desirable cell sources in regenerative dentistry.

Green tea is one of the most commonly consumed drinks around the world. Epigallocatechin3-gallate (EGCG) is the major functional component and the most abundant catechin polyphenol in green tea. EGCG has attracted great attention because it is easy to obtain and has potent 
biological effects, such as anti-inflammatory, antibacterial, antioxidative, and anticarcinogenic activities [9]. Previous studies have reported that EGCG improves the osteogenic differentiation of human bone mesenchymal stem cells (BMSCs) [10], human periodontal ligament cells [11], and human adipose-derived stem cells [12] in vitro. EGCG modified scaffolds enhance osteogenesis and improve bone healing in vivo $[13,14]$. A recent study also revealed that EGCG enhances the odontogenic differentiation of dental pulp stem cells (DPSCs) [15]. According to the abovementioned facts, EGCG seems to be a good agent for promoting osteo-/odontogenic differentiation and mineralized tissue formation. Additionally, EGCG exhibits a great ability to promote the proliferation of human bone mesenchymal stem cells [16] as well as neural stem cells [17,18]. However, the effects of EGCG on the proliferation and osteo-/odontogenic differentiation of SCAPs are unknown.

Odontoblast differentiation and tooth root formation are regulated by several signaling pathways, such as the Wnt signaling pathway and the BMP-Smad signaling pathway [4]. Several BMPs, such as BMP2 and BMP7, are expressed during odontoblast differentiation [19]. Moreover, the controlled release of BMP2 promotes dentin formation from SCAPs in vivo [20]. However, it is unclear whether the BMP-Smad signaling pathway is required for the osteo-/odontogenic differentiation of SCAPs during EGCG treatment.

In this study, we aimed to determine the effects of EGCG on SCAPs by detecting cell proliferation, migration, and odontogenic differentiation, and investigated how EGCG regulates the osteo-/odontogenic differentiation of SCAPs. The null hypothesis of our study was EGCG influences the proliferation, migration, and odontogenic differentiation of SCAPs, and EGCG affects the odontogenic differentiation of SCAPs through BMP signaling. Our results revealed that EGCG can promote the proliferation and osteo-/odontogenic differentiation of SCAPs by activating the BMP-Smad signaling pathway. Furthermore, we found that EGCG showed odontogenic capacity similar to that of recombinant BMP2 in vitro. These findings indicate that EGCG can be a promising natural compound to facilitate dentin regeneration.

\section{Results}

\subsection{In Vitro Characterization of SCAPs}

To obtain SCAPs, we obtained apical papilla from immature human third molars indicated by radiograph (Figure 1A,B) and isolated SCAPs. The isolated cells exhibited colony-like growth after 10 days of culture (Figure $1 \mathrm{C}, \mathrm{D})$. Cells expressed several cell surface markers, such as CD44 (99.06\%), CD90 (99.29\%), CD146 (61.37\%), and CD24 (12.97\%) (Figure 1E), whereas cells were negative for the hematopoietic linage markers CD34 and CD45 (Figure 1E). Additionally, the cells positively expressed the neurogenic marker Nestin, which indicated the SCAPs may be from a neural crest origin of mesenchymal stem cells (MSCs) (Figure 1F). In addition, after induction in adipogenic or osteo-/odontogenic culture medium, the isolated cells formed extensive mineral deposits or lipid clusters, respectively (Figure 1G,H).

\subsection{The Effects of EGCG on Proliferation and Migration of SCAPS}

To determine the effects of EGCG on the proliferation and migration of SCAPs, cells were treated with EGCG at different concentrations. We found that EGCG at concentrations of $0.1-100 \mu \mathrm{M}$ showed no significant effect on the proliferation of SCAPs on day 1. EGCG treatment at 1 or $10 \mu \mathrm{M}$ increased the proliferation of SCAPs at days 3 and 5 when compared with the control group. However, cells showed a significantly lower proliferation ability when treated with 50 or $100 \mu \mathrm{M}$ EGCG after three and five days of culture (Figure 2). We also determined the effect of EGCG on the migration of SCAPs using a wound healing assay. Although higher concentrations of EGCG $(\geq 10 \mu \mathrm{M})$ significantly attenuated the migration of SCAPs, low concentrations of EGCG $(0.1$ or $1 \mu \mathrm{M})$ did not affect the migration of SCAPs after $24 \mathrm{~h}$ of incubation (Figure 3A,B). Based on these results, we used EGCG concentrations no higher than $10 \mu \mathrm{M}$ for the following experiments. 

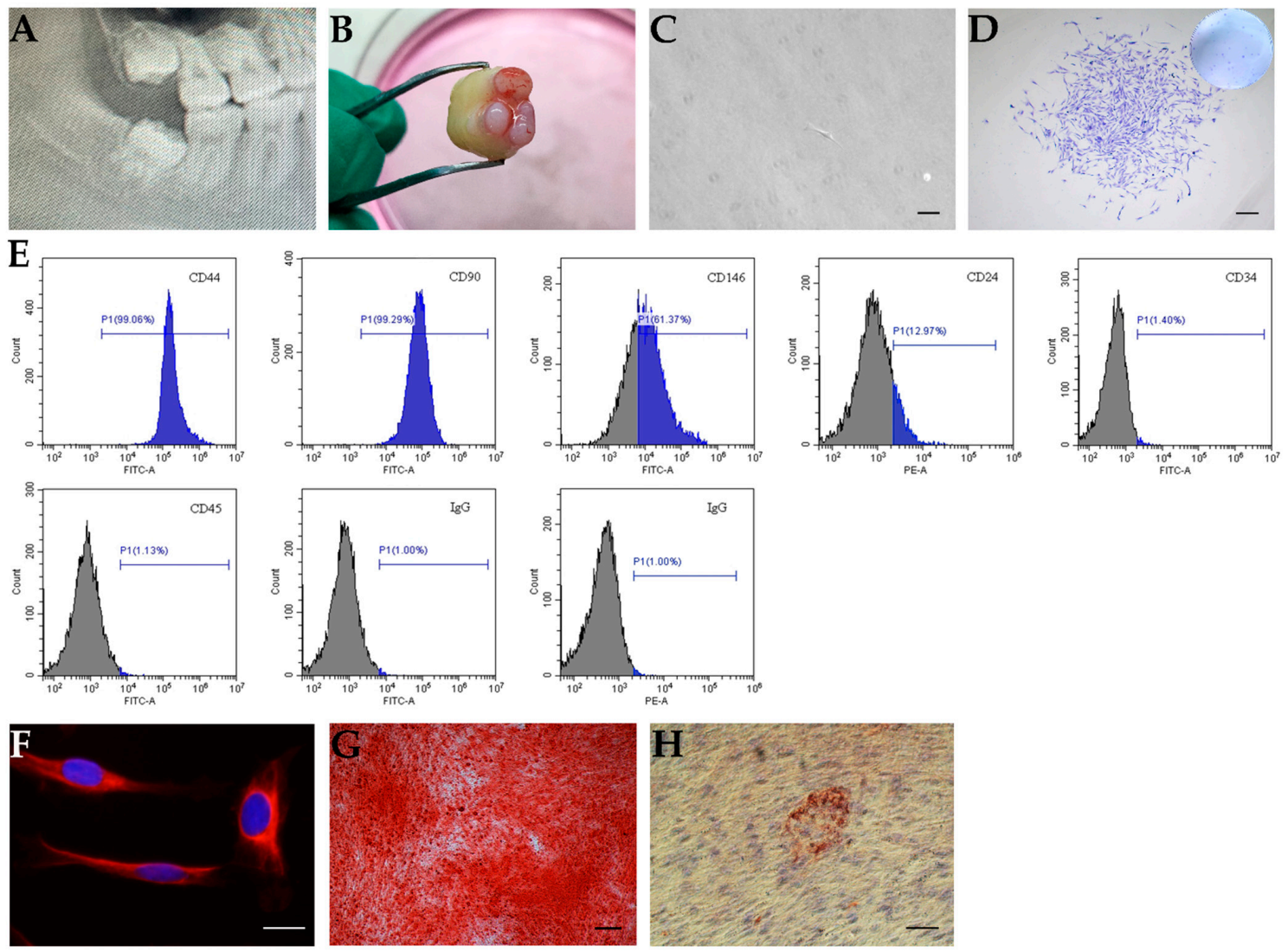

Figure 1. In vitro characterization of stem cells from apical papilla (SCAPs). (A,B) The apical papilla tissue from an immature human tooth. (A) A representative radiograph indicating the third molar with the open apex; (B) apical papilla tissue. (C,D) The colony formation of SCAPs. (C) SCAPs exhibited a typical spindle shape, scale bar $=100 \mu \mathrm{m}$. (D) Isolated cells formed colony-like units, scale bar $=200 \mu \mathrm{m}$. (E) Flow cytometric analysis of SCAPs using several markers. Cells were positive for CD44, CD90, CD146, and CD24, and negative for CD34 and CD45. (F) Immunofluorescence staining showed nestin expression in SCAPs, scale bar $=20 \mu \mathrm{m}$. (G) SCAPs formed mineralized nodules after osteo-/odontogenic differentiation, scale bar $=200 \mu \mathrm{m}$. (H) SCAPs were differentiated into adipocytes with Oil Red O-positive lipid clusters when cultured in adipogenic differentiation medium for four weeks, scale bar $=50 \mu \mathrm{m}$.

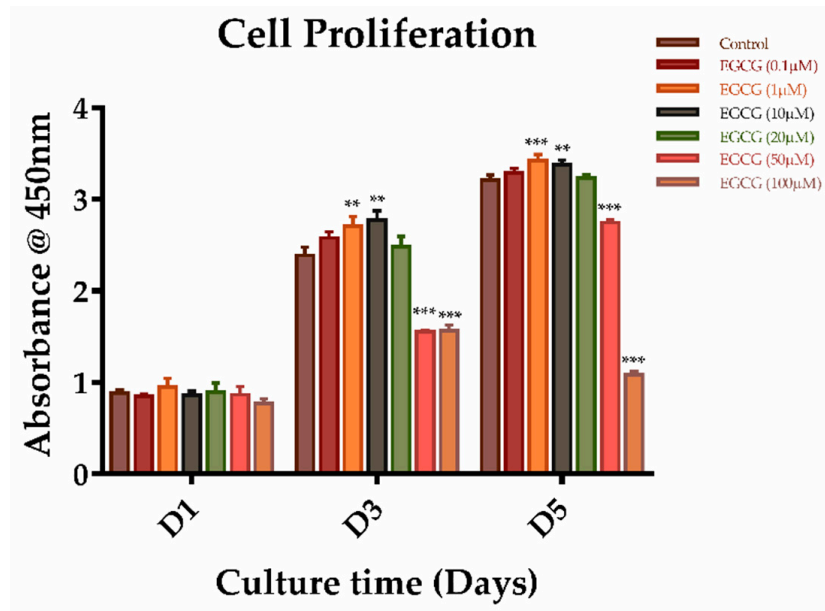

Figure 2. The effects of different concentrations of epigallocatechin-3-gallate (EGCG) on the proliferation of SCAPs. A graph depicting the proliferation of SCAPs treated with different doses of ECGC at days 1,3 , and 5 using a CCK-8 assay $(n=5) .\left({ }^{* *} p<0.01,{ }^{* * *} p<0.001\right)$. Error bars: mean \pm standard deviation. 

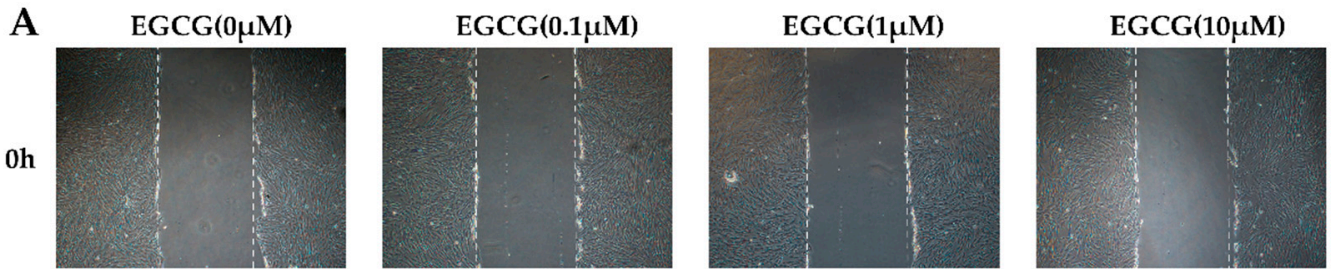

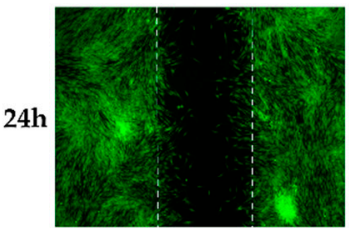

$\operatorname{EGCG}(20 \mu \mathrm{M})$
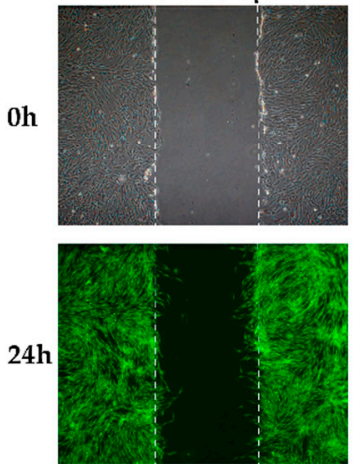

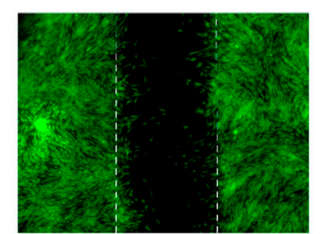

B

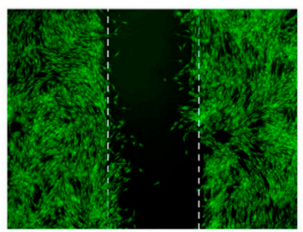

Cell Migration
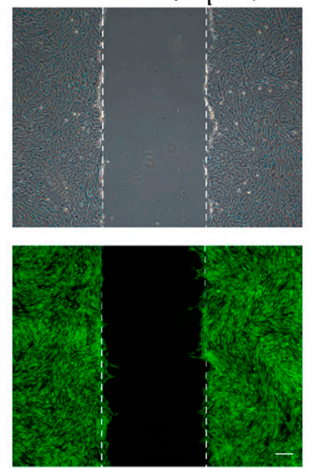

Figure 3. The migration of SCAPs treated with different doses of EGCG. (A) The representative images of the wound-healing assay were obtained at 0 and $24 \mathrm{~h}$ after treatment with different concentrations of EGCG. (B) Migrated cells were counted in each group after $24 \mathrm{~h}$. EGCG at low concentrations exhibited no significant effect compared with the control group, while over $10 \mu \mathrm{M}$ of EGCG inhibited the migration of SCAPs. Scale bar $=200 \mu \mathrm{m} .\left(^{*} p<0.05,{ }^{* * *} p<0.001\right)$. Error bars: mean \pm standard deviation.

\subsection{EGCG Promotes Osteo-/Odontogenic Differentiation and Mineralization of SCAPs}

To evaluate the effect of EGCG on the osteo-/odontogenic differentiation and mineralization of SCAPs, cells were cultured in induction medium containing $0,0.1,1$, or $10 \mu \mathrm{M}$ EGCG for seven or 14 days. After seven days of induction, ALP staining and ALP activity revealed that 1 or $10 \mu \mathrm{M}$ EGCG enhanced the ALP level of SCAPs (Figure 4A,B).

Then, extracellular mineral deposits were determined by ARS staining (Figure 4C) and semi-quantitative analysis (Figure 4D) after 14 days of induction. We determined that treatment with 1 or $10 \mu \mathrm{M}$ EGCG significantly promoted the formation of extracellular mineral deposits, although cells treated with $0.1 \mu \mathrm{M}$ EGCG exhibited comparable results with the control group. Dentin sialophosphoprotein (Dspp), one of the critical odontogenic markers, was significantly enhanced in EGCG-treated groups on day 14 compared with the control group (Figure 4F). With the addition of EGCG, the mRNA level of dentin matrix protein-1 (Dmp-1) and type I collagen (Col 1) was also elevated. EGCG treatment at 1 or $10 \mu \mathrm{M}$ significantly enhanced the expression levels of bone sialoprotein (Bsp), although the $0.1 \mu \mathrm{M}$ EGCG-treated group showed an effect on Bsp expression that was comparable with the control group after 14 days of induction. Cells treated with $10 \mu \mathrm{M}$ EGCG exhibited the most remarkable increases in the expression levels of Dspp, Bsp, and Col 1 on day 14. These changes were further confirmed by western blot analysis. As shown in Figure $4 \mathrm{~F}$, the protein levels of osteo-/odontogenic markers DSPP and DMP-1 were significantly enhanced via EGCG treatment. Collectively, these results indicated that 1 or $10 \mu \mathrm{M}$ EGCG promotes the osteo-/odontogenic differentiation and mineralization of SCAPs. 
A

$A_{\text {EGCG } 0 \mu \mathrm{M}}$

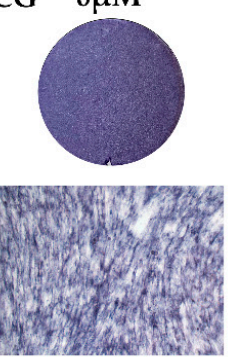

C

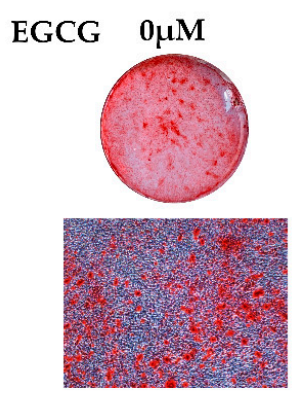

E

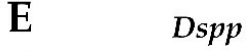

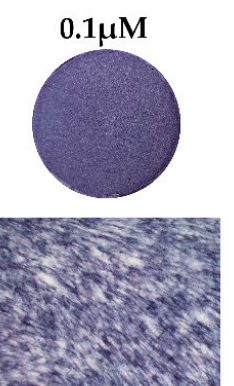
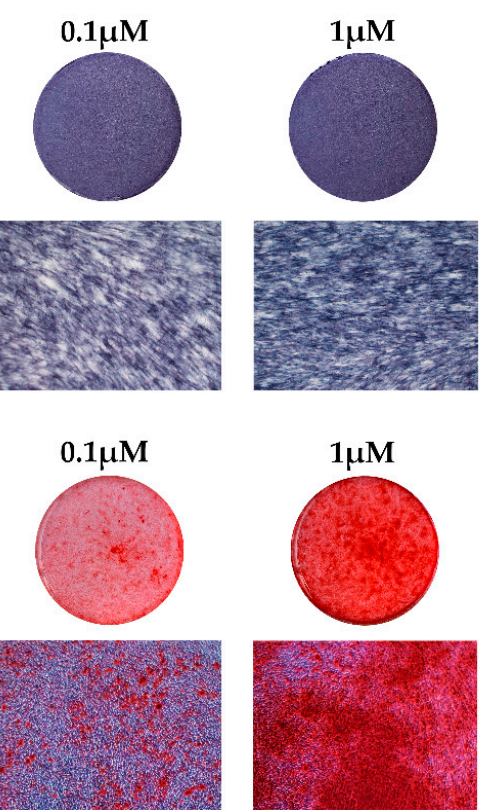

$1 \mu \mathrm{M}$
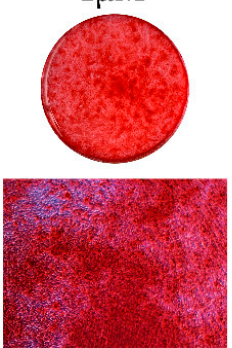

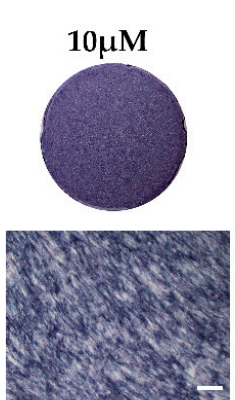

Dmp-1

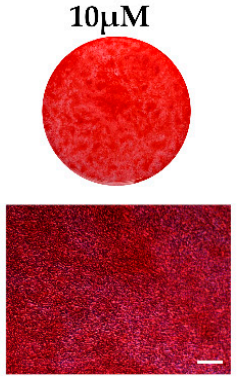

$B s p$
B

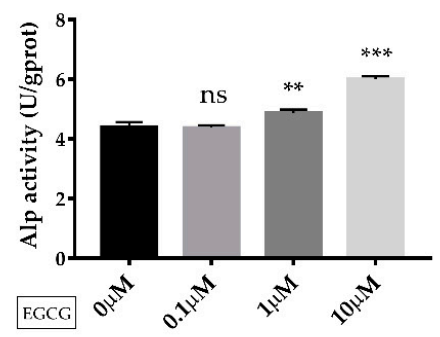

D

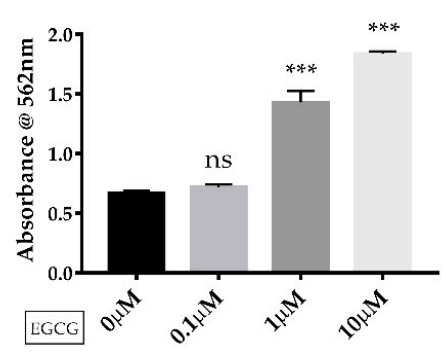

Col1
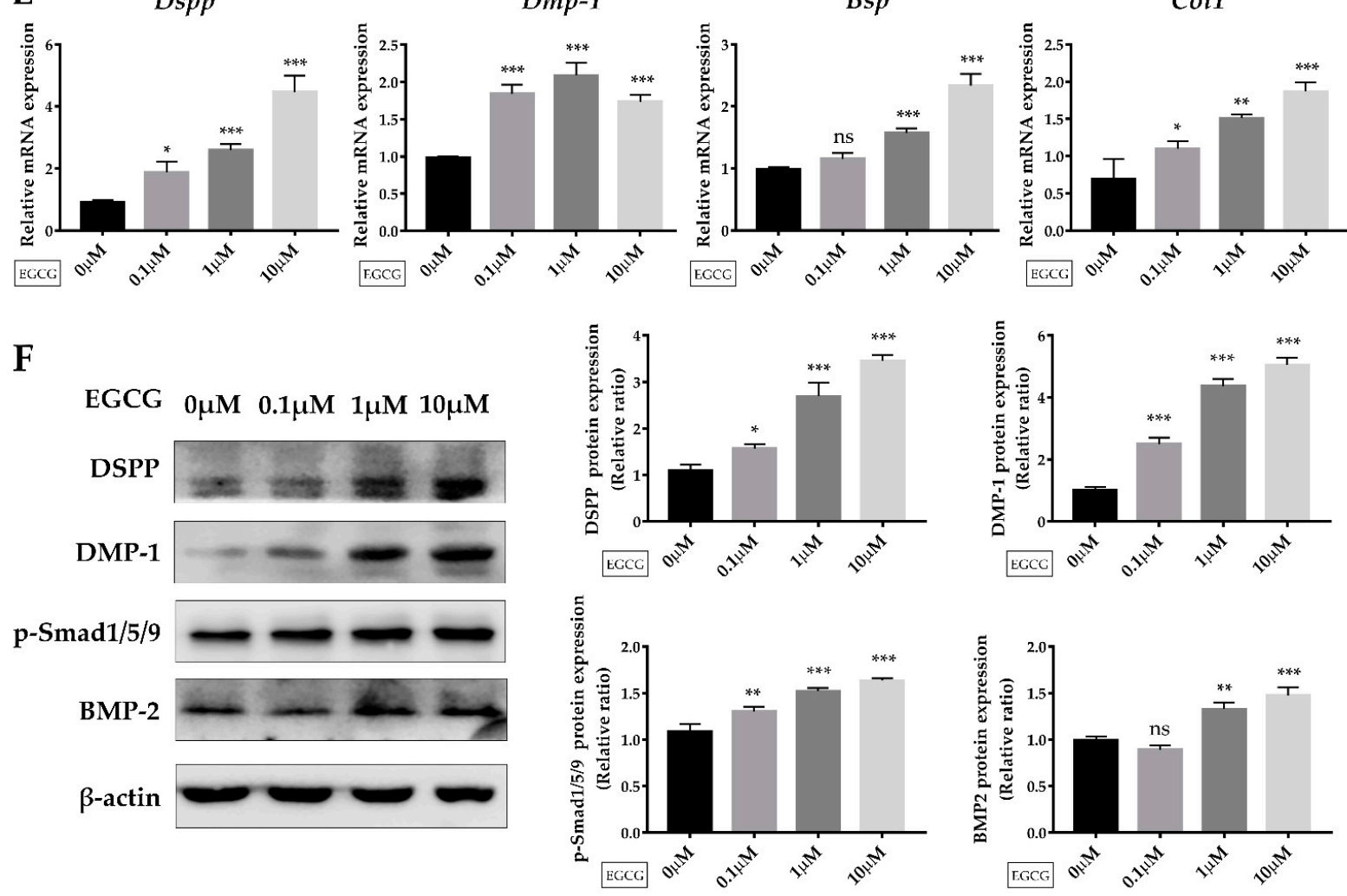

Figure 4. The role of EGCG on the osteo/odontogenic differentiation of SCAPs. (A) ALP staining of SCAPs in each group after seven days of osteo-/odontogenic induction. (B) ALPase activity results showed the upregulation of ALP in EGCG-treated groups at day 7. (C) Alizarin red S staining indicated the formation of mineralized nodules after 14 days of osteo-/odontogenic induction. (D) Semi-quantification of calcium deposits after 14 days of induction. (E) The expression of Dspp, Dmp-1, Bsp, and Col1 was detected at 14 days $(n=3)$. (F) The protein expression and grayscale analysis of DSPP, DMP-1, and BMP2 and the phosphorylation level of Smad1/5/9 at 14 days. Scale bar $=100 \mu \mathrm{m} .{ }^{*} p<0.05,{ }^{* *} p<0.01$, *** $p<0.001$, ns: not statistically significant). Error bars: mean \pm standard deviation. Dspp: dentin sialophosphoprotein; Dmp-1: dentin matrix protein-1; Bsp: bone sialoprotein; Col1: Type I collagen. 


\subsection{Suppression of the BMP-Smad Signaling Pathway Reverses EGCG-Induced Osteo-/Odontogenic Differentiation of SCAPS}

Several studies have reported that BMP-Smad signaling is critically involved in the regulation of the differentiation of MSCs [21,22]. Canonical BMP-Smad signaling leads to the induction of downstream transcription factors and odontogenic differentiation during root initiation and elongation [22,23]. Interestingly, in our study, the expression of BMP-2 as well as p-Smad1/5/9, which are two vital molecules in the BMP-Smad signaling pathway, were upregulated when EGCG was added, suggesting the potential role of the BMP-Smad signaling pathway in EGCG-mediated differentiation of SCAPs (Figure 4F).

Therefore, in order to determine whether the BMP-Smad signaling pathway was affected by EGCG treatment, SCAPs were treated with EGCG for $0.25,0.5,1,2,6$, 12, or $24 \mathrm{~h}$, respectively. p-Smad1/5/9, the important effector of BMP-Smad signaling, was significantly upregulated in SCAPs after EGCG treatment for at least $0.5 \mathrm{~h}$ (Figure 5A). These findings indicate that the BMP-Smad signaling pathway may be the downstream mechanism involved in the EGCG-induced osteo-/odontogenic differentiation of SCAPs.

To confirm whether BMP-Smad signaling is involved in the EGCG-induced osteo/odontogenic differentiation of SCAPs, we employed LDN193189, an inhibitor of type I BMP receptor, to suppress BMP-Smad signaling. As shown in Figure 5B, LDN193189 $(500 \mathrm{nM})$ treatment significantly suppressed the elevated phosphorylation level of Smad1/5/9 caused by EGCG stimulation. Notably, immunofluorescence staining identified the translocation of $\mathrm{p}-\mathrm{Smad} 1 / 5 / 9$ from the cytoplasm into the nucleus with the stimulation of EGCG. However, the nuclear localization of p-Smad1/5/9 was decreased in the EGCG+LDN193189 group compared with the EGCG group (Figure 5C). Furthermore, LDN193189 significantly abrogated the effects of EGCG on the osteo-/odontogenic differentiation of SCAPs. After 14 days of osteo-/odontogenic differentiation, LDN 193,189 reversed EGCG-induced mineralized nodule formation, as indicated by ARS staining (Figure 5D,E). The expression levels of Dspp, Dmp-1, and Col 1 were remarkably decreased in the EGCG+LDN193189 group compared with the EGCG group (Figure 5F). In addition, the protein levels of DSPP and DMP-1 showed a similar tendency to gene expression levels in SCAPs treated with LDN193189 (Figure 5G). Based on these results, we determined that BMP-Smad signaling is required for the EGCG-induced osteo-/odontogenic differentiation of SCAPs.

\subsection{EGCG Shows the Comparable Ability to Promote Mineralization with Recombinant BMP2}

BMP2 has shown a strong and reliable effect in promoting the osteo-/odontogenic differentiation of several kinds of stem cells, both in vitro and in vivo [20]. To facilitate a comprehensive understanding of the ability of EGCG to promote odontogenic differentiation, we compared the effective level of EGCG in osteo-/odontogenic differentiation and mineralization of SCAPs with that of BMP2 in vitro. Cells were treated with EGCG $(1 \mu \mathrm{M})$ or recombinant BMP2 $(10,50 \mathrm{ng} / \mathrm{mL})$. ARS staining indicated the mineralization of the EGCG-treated group had a comparable result with the $50 \mathrm{ng} / \mathrm{mL}$ BMP-treated group after 14 days of induction (Figure 6A,B). The RNA levels of Dspp showed expression levels comparable with the $50 \mathrm{ng} / \mathrm{mL}$ BMP-treated group, while Dmp-1 exhibited a similar change between the EGCG-treated group and $10 \mathrm{ng} / \mathrm{mL}$ BMP-treated group (Figure 6C). These results suggested the EGCG might possess an ability to promote mineralization comparable with that of BMP2. 
A

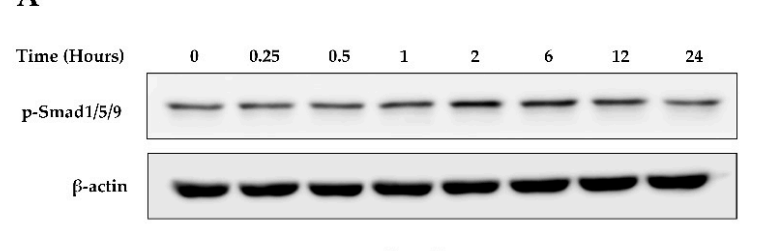

B

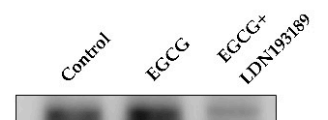

p-Smad1/5/9

$\beta$-actin
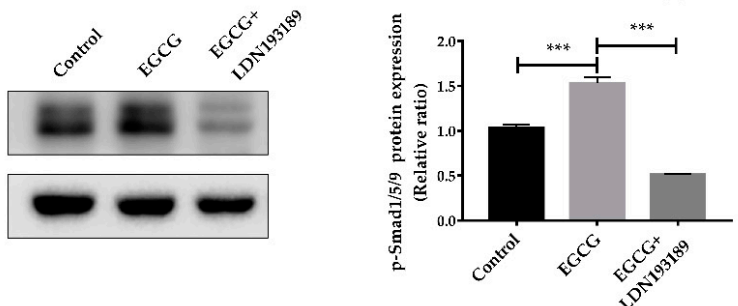

C

Control
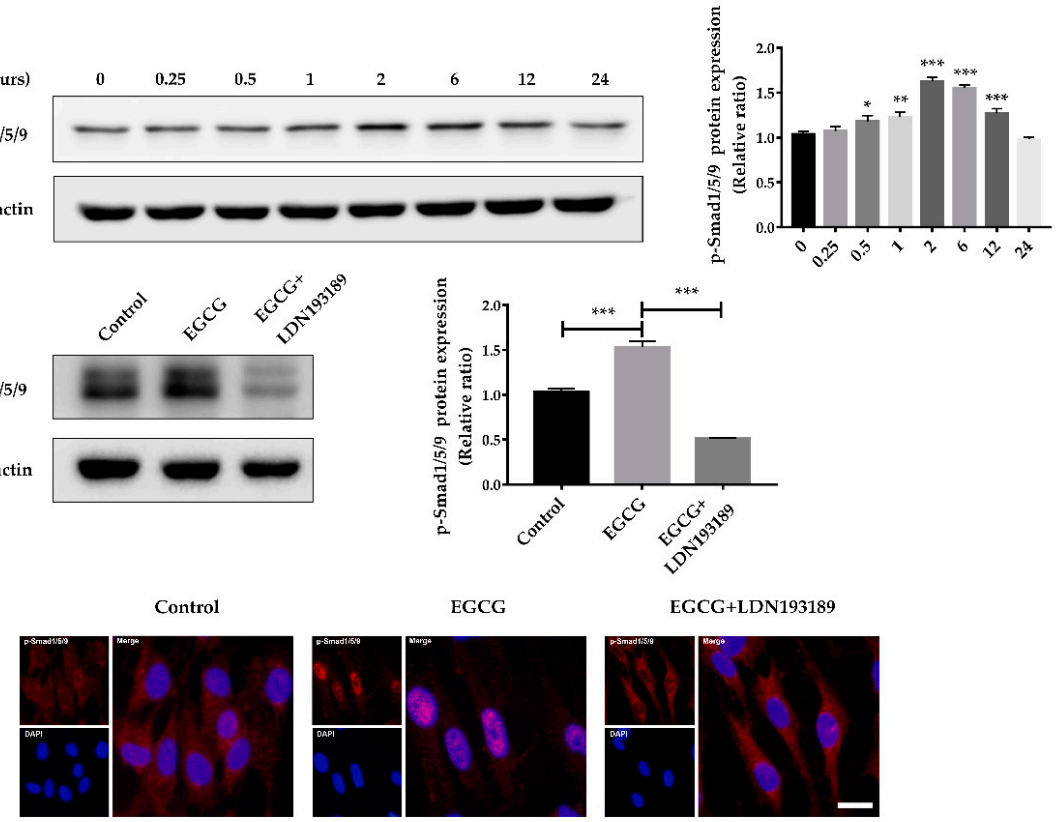

EGCG

EGCG+LDN193189

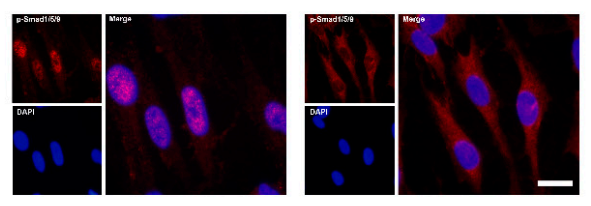

D

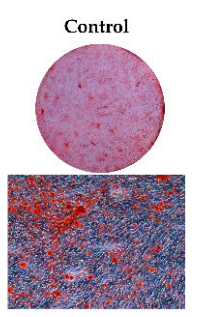

EGCG

EGCG+LDN193189

E
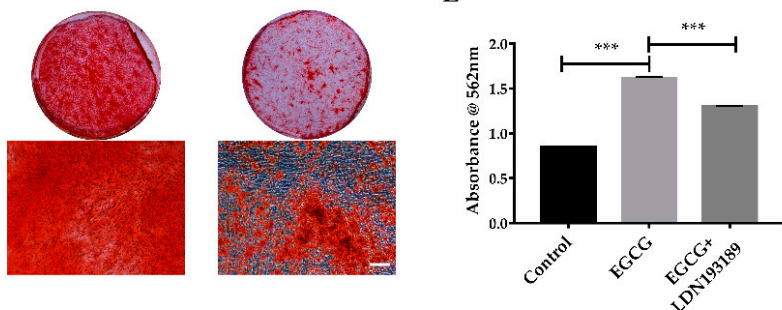

F

$D_{s p p}$

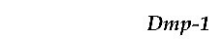

mp-1
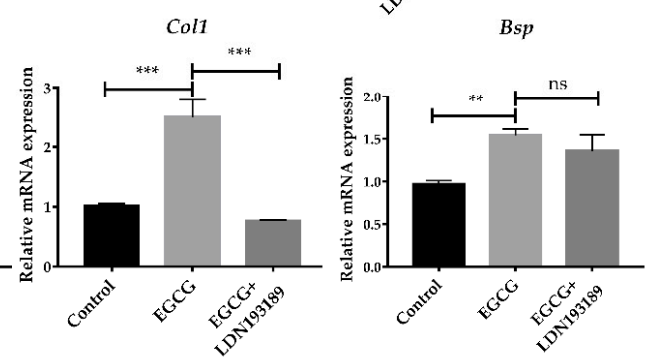

G

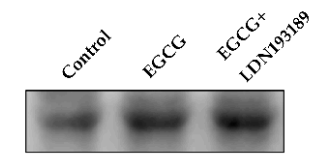

DMP-1

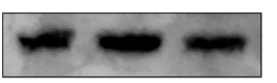

$\beta$-actin
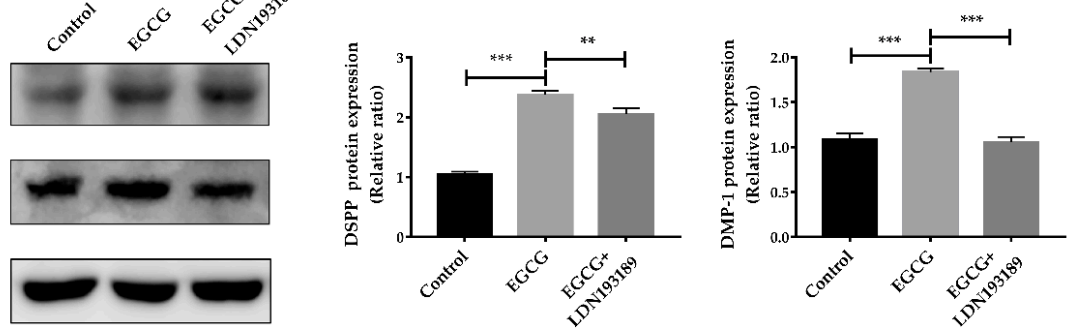

Figure 5. The BMP-Smad signaling pathway is involved in EGCG-stimulated osteo-/odontogenic differentiation. (A) The phosphorylation level of Smad1/5/9 in SCAPs treated with EGCG at different time points and the quantitative analysis. (B) Western blot analysis showing the increased phosphorylation of Smad1/5/9 was reversed by LDN193189. (C) Immunofluorescence staining indicating EGCG increased the translocation of p-Smad1/5/9 from the cytoplasm into the nucleus, but LDN193189 inhibited this transference. Scale bar $=20 \mu \mathrm{m}$. (D) ARS staining in the control $(0 \mu \mathrm{M})$ group, EGCG $(1 \mu \mathrm{M})$ group, and EGCG+LDN193189 group after 14 days of induction. (E) Semi-quantification of calcium deposits. (F) The gene expression levels of Dspp, Dmp-1,Bsp, and Col1 in inhibitor group (EGCG+LDN193189) were suppressed compared with in the EGCG group $(n=3)$. (G) The protein levels and quantitative analysis of DSPP and DMP-1 at 14 days. Error bars: mean \pm standard deviation. $\left({ }^{*} p<0.05,{ }^{* *} p<0.01,{ }^{* * *} p<0.001\right.$, ns: not statistically significant). 
A

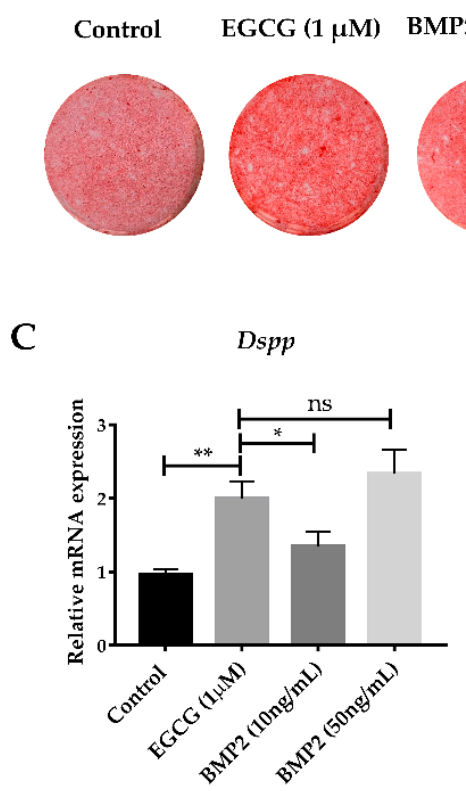

B

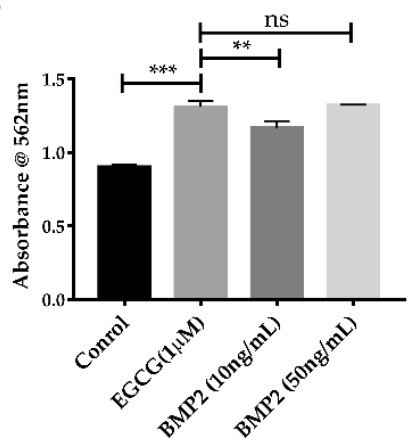

Figure 6. EGCG showed the ability to promote mineralization comparable with that of BMP2. (A) After 14 days of induction, the EGCG $(1 \mu \mathrm{M})$ group generated amounts of calcified nodules similar to the $50 \mathrm{ng} / \mathrm{mL}$ BMP2 group. (B) Semi-quantification of mineralized deposits. (C) RNA expression of Dspp and Dmp-1 at 14 days $(n=3)$. Error bars: mean \pm standard deviation. $\left({ }^{*} p<0.05,{ }^{* *} p<0.01,{ }^{* * *} p<0.001\right.$, ns: not statistically significant).

\section{Discussion}

In our study, we found that EGCG promotes the proliferation of SCAPs while showing no significant influence on cell migration at concentrations below $10 \mu \mathrm{M}$. Meanwhile, treatment with 1 or $10 \mu \mathrm{M}$ EGCG enhances the osteo-/odontogenic differentiation of SCAPs as indicated by ALP activity, mineral deposition, and the expression of osteo-/odontogenic related markers. We further determined that EGCG promotes osteo-/odontogenic differentiation by activating the BMP-Smad signaling pathway in vitro. Finally, we found that EGCG shows an ability to promote mineralization comparable to that of recombinant BMP2. Our findings indicated that EGCG is an efficient natural chemical promoting the osteo-/odontogenic differentiation and mineralization of SCAPs and may be used for regenerative dentistry.

Dental tissue engineering requires stem/progenitor cells. Among the mesenchymal stem cells identified from dental tissues, SCAPs derived from the apex of an immature tooth may be a superior source for dentin regeneration due to their origin. In our study, we isolated and characterized human SCAPs, as the cells showed colony formation ability, multilineage differentiation potential, and the expression of mesenchymal stem cell markers (e.g., CD44, CD90, and CD146). Meanwhile, CD24, which might be a specific marker of SCAPs [6], can also be detected in the cells we isolated. However, the percentage of cells expressing CD24 shows a wide range [6,24]. This may be related with the donors or the cell condition, etc. SCAPs show significantly higher telomerase activity and express greater abundance of survivin than DPSCs [6]. This indicates that SCAPs might be more naive cells. Additionally, it has been documented that SCAPs express more DMP-1 than stem cells from human exfoliated deciduous teeth (SHED) under differentiation conditions, and genomewide gene expression profiles show that SCAPs are precursors for primary odontoblasts, whereas SHED differentiate into replacement odontoblasts [25]. Meanwhile, SCAPs have a higher population doubling capacity along with higher proliferation and migration capacity when compared with DPSCs [6,26]. SCAPs also show higher mineralization potential and dentin regeneration capacity than DPSCs in vitro [26] and in vivo [6]. These important advantages make SCAPs a promising source for dental tissue regeneration. 
In order to promote the efficiency of tissue regeneration, various molecules are used. In our study, we determined the dual effect of EGCG on the proliferation of SCAPs, that is, low concentrations of EGCG promote cell proliferation, while high concentrations of EGCG show an inhibited effect. Several studies have demonstrated that EGCG could promote the proliferation of cells such as PDLCs and human BMSCs in vitro at concentrations below $20 \mu \mathrm{M}$ [11,12], which is consistent with our results. Differences in cell types, culture conditions, or detection methods may be accountable for the discrepancies in concentrations of EGCG. Nevertheless, previous studies have shown that higher concentrations of EGCG significantly suppress cell proliferation in cancer cells $[27,28]$ and adult stem cells [16], similarly to our results. The inhibitive effect of EGCG at high concentrations may be related to apoptotic cell death via DNA damage and reduction of DNA synthesis [29]. On the other hand, cell migration has also been shown to be important in regenerative dentistry. High concentrations ( 25 and $50 \mu \mathrm{M}$ ) of EGCG may reduce the migration of human alveolar bone cells [30]. Therefore, a combination of molecules that promote migration like SDF- $1 \alpha$ might be an option when we use higher concentrations of EGCG. Collectively, our findings showed that EGCG can promote the proliferation of SCAPs, and low concentrations ( 0.1 or $1 \mu \mathrm{M})$ of EGCG do not have a significant influence on cell migration. Therefore, $1-10 \mu \mathrm{M}$ of EGCG may be the recommended concentration for future applications.

The regeneration of dentin involves the odontogenic differentiation of stem cells and the formation of mineralized tissues. Our results revealed that EGCG enhances the expression of osteo-/odontogenic markers including BSP and Col 1 in SCAPs. Previous research reported that EGCG increases the expression of Col1 and osterix (Osx) and promotes extracellular matrix mineralization in PDLCs [11]. Furthermore, several studies have found EGCG can enhance ALP activity, the expression of osteogenic markers like Runx2 and OCN, and the formation of mineralized nodules $[10,11,31]$. However, the effective concentrations for osteogenic differentiation varied among studies, which might be related to the cell types. Moreover, DMP-1 and DSPP, the two major phosphoproteins of non-collagenous proteins, can regulate dentin mineralization. In our study, these two representative odontogenic markers were also upregulated when EGCG was added. In accordance with previous studies, our findings indicate that EGCG can promote the osteo-/odontogenic differentiation and mineralization of SCAPs.

Although BMPs show a potent capacity to promote odontogenic differentiation and dentin regeneration, the large dose requirement and high cost are obstacles for clinical applications. It has been reported that an increase in BMP-2 has been identified in the EGCG-induced osteogenic differentiation of BMSCs [10]. In our study, we determined that EGCG increases the expression of BMP2 and the phosphorylation level of Smad1/5/9, and the suppression of BMP-Smad signaling using LDN193189 significantly attenuates the EGCG-induced osteo-/odontogenic differentiation of SCAPs. Moreover, we compared the effects of BMP2 and EGCG on the osteo-/odontogenic differentiation of SCAPs and determined the concentrations of BMP2 and EGCG, which, to some extent, exhibited similar capacities. Furthermore, recent studies reported that an EGCG-modified scaffold exhibited desirable morphological and mechanical characteristics, as well as having an anti-inflammatory effect, and could modulate the recruitment of cells [32-34]. EGCG is, therefore, a potential therapeutic molecule for tissue regeneration when incorporated with a scaffold or other agents. Further work using such an EGCG-modified scaffold with SCAPs in an animal model is still needed.

\section{Materials and Methods}

\subsection{Cell Culture}

For the harvesting of SCAPs, healthy immature human third molars were collected from patients aged 16-20 years $(n=5)$ according to the previous studies [6]. All protocols in the present work were approved by the Ethics Committee of the School of Stomatology, Wuhan University, China. In brief, apical papilla tissues were gently separated from teeth, thoroughly rinsed with phosphate buffered saline (PBS, Hyclone, Logan, UT), minced, 
and digested with $3 \mathrm{mg} / \mathrm{mL}$ type I collagenase (Biosharp, Hefei, China) and $4 \mathrm{mg} / \mathrm{mL}$ dispase (Roche, Mannheim, Germany) at $37^{\circ} \mathrm{C}$ for $40 \mathrm{~min}$. The digested mixtures were passed through a $70 \mu \mathrm{m}$ cell strainer to get single-cell suspensions. SCAPs were cultured in the $\alpha$-MEM (Hyclone) containing 10\% fetal bovine serum (FBS, Gibco, Thornton, NSW, Australia) and $100 \mathrm{U} / \mathrm{mL}$ penicillin-streptomycin (Hyclone). Cells were incubated at $37^{\circ} \mathrm{C}$ in an atmosphere with $5 \% \mathrm{CO}_{2}$. SCAPs at passages 2 to 5 were used in this study.

\subsection{Colony-Forming Assay}

To characterize SCAPs, single-cell suspensions of SCAPs were seeded in a $10 \mathrm{~cm}$ dish. Cells were cultured in the culture medium for 10 days. After fixation in $4 \%$ paraformaldehyde (PFA), cells were immersed in crystal violet staining solution for 10 min (Beyotime, Shanghai, China). Clusters with more than 50 cells were considered as colonies.

\subsection{Flow Cytometric Analysis}

SCAP aliquots $\left(1 \times 10^{6}\right.$ cells $)$ were used to detect the immunophenotype according to a previously developed protocol [35]. The following anti-human monoclonal antibodies were used: CD45/FITC (Cat\#304005, BioLegend, San Diego, CA, USA), CD34/FITC (Cat\# 343603, BioLegend), CD90/FITC (Cat\#328107, BioLegend), CD146/FITC (Cat\#361011, BioLegend), CD44/FITC (Cat\#338803, BioLegend), and CD24/PE (Cat\#311105, BioLegend). Non-specific IgG/FITC (Cat\#400107, BioLegend) or IgG/PE (Cat\#400211, BioLegend) was used to be the isotype control. The data were analyzed using a FACS Calibur flow cytometer (Becton Dickinson, Franklin Lakes, NJ, USA).

\subsection{Immunofluorescence Staining}

For immunofluorescent analyses, cells were seeded on coverslips. After washing and fixation, cells at passage 2 were incubated with anti-Nestin (1:100, Santa Cruz Biotechnology, Santa Cruz, CA, USA), and cells treated with EGCG alone or EGCG combined with LDN193189 were incubated with anti-p-Smad1/5/9 (1:500, Cell Signaling Technology, Beverly, MA, USA) overnight. After one hour of incubation with Cy3-conjugated secondary antibodies (Jackson ImmunoResearch, West Grove, PE, USA), samples were counterstained with 4,6-diamidino-2-phenylindole (DAPI). Then, cells were photographed under a fluorescence microscope (Leica, Wetzlar, Germany).

\subsection{Multiple Lineage Differentiation}

For osteo-/odontogenic differentiation, SCAPs were cultured in six-well plates. The osteo-/odontogenic differentiation induction medium was supplied for three weeks. The induction medium contained $\alpha$-MEM with $10 \%$ FBS, $50 \mathrm{mg} / \mathrm{mL}$ ascorbic acid, $10 \mathrm{mM}$ sodium $\beta$-glycerophosphate, and $10 \mathrm{nM}$ dexamethasone (Sigma-Aldrich, St. Louis, MI, USA) [36]. Cells were fixed with 95\% ethanol, and then washed and stained with $1 \%$ Alizarin Red S (Sigma-Aldrich; $\mathrm{pH}=4.2$ ) in order to detect the formation of mineral deposits.

For adipogenic differentiation, cells were cultured in adipogenic medium (Cyagen Biosciences Inc, Sunnyvale, CA, USA) according to the manufacturer's instructions. After four weeks, cells were fixed in 4\% PFA. Next, Oil Red O reagent (Cyagen) was used to stain cells for $15 \mathrm{~min}$ to observe lipid droplets.

\subsection{CCK-8 Assay}

For the CCK-8 assay, $5 \times 10^{3}$ SCAPs per well were seeded in 96-well plates and incubated with culture medium containing 0, 0.1, 1, 10, 20, 50, or $100 \mu \mathrm{M}$ EGCG (SigmaAldrich, \#E4143). The culture medium was renewed every other day. The proliferation of the SCAPs was measured with a CCK-8 assay kit (Dojindo, Kumamoto, Japan) after one, three, or five days of culture following the manufacturer's instructions. 


\subsection{Wound Healing Assay}

Wound healing assay was performed to assess the role of EGCG on the motility of SCAPs in vitro [8]. SCAPs were seeded in six-well plates, and the monolayers were scratched using a $200 \mu \mathrm{L}$ pipette tip. PBS washed twice to remove cell debris. Cells were maintained in $\alpha$-MEM containing $1 \%$ FBS and different concentrations of EGCG $(0,0.1,1,10,20$, or $50 \mu \mathrm{M})$. Images were captured immediately after scratching under a phase contrast microscope (Olympus, Tokyo, Japan). After $24 \mathrm{~h}$, cells were fixed with $4 \%$ PFA. Then, cells were incubated with $0.1 \mathrm{mg} / \mathrm{mL}$ acridine orange and visualized under a fluorescent microscope (Olympus). Cells from five random areas were used to calculate the average amount of migrating cells in each group.

\subsection{Alkaline Phosphatase (ALP) Staining and Alizarin Red S (ARS) Staining}

Cells were cultured in 12-well plates supplied with osteo-/odontogenic differentiation induction medium. In different groups, EGCG was added into the medium at concentrations of $0,0.1,1$, or $10 \mu \mathrm{M}$. The medium was renewed every three days. For ALP staining, after seven days of osteo-/odontogenic induction, cells from each group were fixed with $4 \%$ PFA. Then, cells were stained with CIP/NBT Alkaline Phosphatase Color Development Kit (Beyotime). The quantitation of ALP activity was detected using an Alkaline Phosphatase Assay Kit (Nanjing Jiancheng Bioengineering Institute, Nanjing, China) according to the manufacturer's instructions. The quantitative values were measured at $520 \mathrm{~nm}$ absorbance. The values of ALP activity were calculated in U/gprot.

For ARS staining, cells were stained with $1 \%$ Alizarin Red S after 14 days of osteo/odontogenic induction. For semi-quantification of ARS staining, 10\% $(w / v)$ cetylpyridinium chloride was added to the plates to destain the samples. The absorbance was determined at $562 \mathrm{~nm}$ using a microplate reader.

\subsection{Quantitative Reverse Transcriptase Polymerase Chain Reaction ( $q R T-P C R$ ) Analysis}

The mRNA expression levels of Dspp, Dmp-1, Bsp, and Col1 were determined via qRTPCR. Briefly, total RNAs were isolated from SCAPs with the HP Total RNA Kit (Omega bio-tech, Norcross, GA, USA) after seven or 14 days of osteo-/odontogenic induction. Then, a Revert Aid First Strand cDNA Synthesis Kit (ThermoFisher Scientific, Rockford, IL, USA) was used to synthesize cDNA. The cDNA was then used for qRT-PCR in a CFX Connect Real-Time System (Bio-rad, Hercules, CA, USA) with SYBR Green Master (Roche). The expression levels of the target genes were normalized to GAPDH using the $2^{-\Delta \Delta C t}$ method. The primers for qRT-PCR are listed in Table 1.

Table 1. Primers for qRT-PCR.

\begin{tabular}{|c|c|c|}
\hline Genes & Forward Primer $\left(5^{\prime}-3^{\prime}\right)$ & Reverse Primer $\left(5^{\prime}-3^{\prime}\right)$ \\
\hline Gapdh & TCATGGGTGTGAACCATGAGAA & GGCATGGACTGTGGTCATGAG \\
\hline$D s p p$ & TGCTGGAGCCACAAAC & AAACCCTATGCAACСТTC \\
\hline Dmp-1 & ACAGGCAAATGAAGACCC & TTCACTGGCTTGTATGG \\
\hline Bsp & CGAAGCAGAAGTGGATGAAA & TGCCTCTGTGCTGTTGGTACTG \\
\hline Col1 & GCGGCTCCCCATTTTTATACC & GCTCTCCTCCCATGTTAAATAGCAC \\
\hline
\end{tabular}

Gapdh: glyceraldehyde-3-phosphate dehydrogenase; Dspp: dentin sialophosphoprotein; Dmp-1: dentin matrix protein-1; Bsp: bone sialoprotein; Col1: Type I collagen.

\subsection{Western Blotting}

SCAPs treated with EGCG, LDN193189 (Sigma-Aldrich), or recombinant human BMP2 (PeproTech, Rocky Hill, NJ) at different time points were lysed in lysis buffer (Beyotime), and the protease inhibitor cocktail (MCE, Shanghai, China) was added. After centrifuging at $12,000 \mathrm{rpm}$ for $10 \mathrm{~min}$ at $4{ }^{\circ} \mathrm{C}$, total protein was collected from the lytic cells. The concentrations of proteins were measured using a BCA Kit (Thermo). Equal amounts of proteins were loaded onto $10 \%$ SDS-polyacrylamide gel. The samples were separated by electrophoresis and then transferred onto PVDF membranes (Roche Diagnostics $\mathrm{GmbH}$, 
Germany). The following primary antibodies were used: DSPP (Novus, Centennial, Colorado; Cat\#NBP1-91612, 1:1000), DMP-1 (Abcam, Cambridge, UK; Cat\#103203, 1:1000), p-Smad1/5/9 (Cell Signaling Technology; Cat\#13820, 1:1000), $\beta$-actin (MBL, Tokyo, Japan; Cat\#PM053-7, 1:6000), and BMP2 (Abcam; Cat\#6285, 1:1000). The target bands were quantified using Image $\mathrm{J}$ and normalized to $\beta$-actin.

\subsection{Statistical Analysis}

The results shown in mean \pm standard deviation were representative of three independent experiments. The equal variance between individual groups showed no significant difference. The data in the study were analyzed using one-way analysis of variance (ANOVA), followed by Tukey's multiple post hoc tests using GraphPad Prism 7.0 (GraphPad Software, La Jolla, CA, USA). $p<0.05$ was considered statistically significant.

\section{Conclusions}

Based on the findings of our study, we found that EGCG promotes the proliferation of SCAPs, and low doses $(0.1$ or $1 \mu \mathrm{M})$ of EGCG do not influence the migration of SCAPs. EGCG increases the osteo-/odontogenic differentiation of SCAPs by activating the BMP-Smad signaling pathway. EGCG may possess a comparable ability to promote mineralization with recombinant BMP2 in vitro. Our results suggest EGCG can be used as an effective natural compound in dentin regeneration.

Author Contributions: Conceptualization: Z.L., J.Y., and Z.C.; methodology: Z.L. and Y.L.; formal analysis: Z.L., Y.L., and X.F.; resources: Z.L. and X.F.; funding acquisition: J.Y and Z.C.; writingoriginal draft preparation: Z.L.; writing—review and editing: J.Y and Z.C.; supervision: J.Y and Z.C.; All authors have read and agreed to the published version of the manuscript.

Funding: This research was funded by grants from National Natural Science Foundation of China (No. 81771066) and Young Scientists Fund of National Natural Science Foundation of China (No. 31500788).

Institutional Review Board Statement: The study was conducted according to the guidelines of the Declaration of Helsinki, and approved by the Ethics Committee of School of Stomatology, Wuhan University, China.

Informed Consent Statement: Not applicable.

Data Availability Statement: The data presented in this study are available included in the article.

Acknowledgments: The author thanks the Wuhan University School and Hospital of stomatology for its support to the experimental conditions.

Conflicts of Interest: The authors declare no conflict of interest.

Sample Availability: Not available.

\section{References}

1. Xuan, K.; Li, B.; Guo, H.; Sun, W.; Kou, X.; He, X.; Zhang, Y.; Sun, J.; Liu, A.; Liao, L.; et al. Deciduous autologous tooth stem cells regenerate dental pulp after implantation into injured teeth. Sci. Transl. Med. 2018, 10. [CrossRef] [PubMed]

2. Palma, P.J.; Ramos, J.C.; Martins, J.B.; Diogenes, A.; Figueiredo, M.H.; Ferreira, P.; Viegas, C.; Santos, J.M. Histologic Evaluation of Regenerative Endodontic Procedures with the Use of Chitosan Scaffolds in Immature Dog Teeth with Apical Periodontitis. J. Endod. 2017, 43, 1279-1287. [CrossRef] [PubMed]

3. Palma, P.J.M.J.; Diogo, P.; Sequeira, D.; Ramos, J.C.; Diogenes, A.; Santos, J.M. Does Apical Papilla Survive and Develop in Apical Periodontitis Presence after Regenerative Endodontic Procedures? Appl. Sci. 2019, 9, 3942. [CrossRef]

4. Li, J.; Parada, C.; Chai, Y. Cellular and molecular mechanisms of tooth root development. Development 2017, 144, 374-384. [CrossRef]

5. Huang, G.T.; Sonoyama, W.; Liu, Y.; Liu, H.; Wang, S.; Shi, S. The hidden treasure in apical papilla: The potential role in pulp/dentin regeneration and bioroot engineering. J. Endod. 2008, 34, 645-651. [CrossRef]

6. Sonoyama, W.; Liu, Y.; Fang, D.; Yamaza, T.; Seo, B.M.; Zhang, C.; Liu, H.; Gronthos, S.; Wang, C.Y.; Wang, S.; et al. Mesenchymal stem cell-mediated functional tooth regeneration in swine. PLoS ONE 2006, 1, e79. [CrossRef] 
7. Dong, R.; Yao, R.; Du, J.; Wang, S.; Fan, Z. Depletion of histone demethylase KDM2A enhanced the adipogenic and chondrogenic differentiation potentials of stem cells from apical papilla. Exp. Cell Res. 2013, 319, 2874-2882. [CrossRef]

8. Sequeira, D.B.; Seabra, C.M.; Palma, P.J.; Cardoso, A.L.; Peça, J.; Santos, J.M. Effects of a New Bioceramic Material on Human Apical Papilla Cells. J. Funct. Biomater. 2018, 9, 74. [CrossRef]

9. Chowdhury, A.; Sarkar, J.; Chakraborti, T.; Pramanik, P.K.; Chakraborti, S. Protective role of epigallocatechin-3-gallate in health and disease: A perspective. Biomed. Pharmacother. 2016, 78, 50-59. [CrossRef] [PubMed]

10. Lin, S.Y.; Kang, L.; Wang, C.Z.; Huang, H.H.; Cheng, T.L.; Huang, H.T.; Lee, M.J.; Lin, Y.S.; Ho, M.L.; Wang, G.J.; et al. (-)Epigallocatechin-3-Gallate (EGCG) Enhances Osteogenic Differentiation of Human Bone Marrow Mesenchymal Stem Cells. Molecules 2018, 23, 3221. [CrossRef] [PubMed]

11. Liu, J.; Lu, Y.; Liu, J.; Jin, C.; Meng, Y.; Pei, D. Influence of epigallocatechin-3-gallate in promoting proliferation and osteogenic differentiation of human periodontal ligament cells. BMC Oral Health 2019, 19, 73. [CrossRef]

12. Zhang, J.; Wu, K.; Xu, T.; Wu, J.; Li, P.; Wang, H.; Wu, H.; Wu, G. Epigallocatechin-3-gallate enhances the osteoblastogenic differentiation of human adipose-derived stem cells. Drug Des. Dev. Ther. 2019, 13, 1311-1321. [CrossRef] [PubMed]

13. Sasayama, S.; Hara, T.; Tanaka, T.; Honda, Y.; Baba, S. Osteogenesis of Multipotent Progenitor Cells using the Epigallocatechin Gallate-Modified Gelatin Sponge Scaffold in the Rat Congenital Cleft-Jaw Model. Int. J. Mol. Sci. 2018, 19, 3803. [CrossRef]

14. Shin, Y.S.; Seo, J.Y.; Oh, S.H.; Kim, J.H.; Kim, S.T.; Park, Y.B.; Moon, H.S. The effects of ErhBMP-2-/EGCG-coated BCP bone substitute on dehiscence around dental implants in dogs. Oral Dis. 2014, 20, 281-287. [CrossRef] [PubMed]

15. Kwon, Y.S.; Kim, H.J.; Hwang, Y.C.; Rosa, V.; Yu, M.K.; Min, K.S. Effects of Epigallocatechin Gallate, an Antibacterial Cross-linking Agent, on Proliferation and Differentiation of Human Dental Pulp Cells Cultured in Collagen Scaffolds. J. Endod. 2017, 43, 289-296. [CrossRef]

16. Wang, D.; Wang, Y.; Xu, S.; Wang, F.; Wang, B.; Han, K.; Sun, D.; Li, L. Epigallocatechin-3-gallate Protects against Hydrogen Peroxide-Induced Inhibition of Osteogenic Differentiation of Human Bone Marrow-Derived Mesenchymal Stem Cells. Stem Cells Int. 2016, 2016, 7532798. [CrossRef]

17. Zhang, Y.; He, Q.; Dong, J.; Jia, Z.; Hao, F.; Shan, C. Effects of epigallocatechin-3-gallate on proliferation and differentiation of mouse cochlear neural stem cells: Involvement of PI3K/Akt signaling pathway. Eur. J. Pharm Sci. 2016, 88, 267-273. [CrossRef]

18. Wang, Y.; Li, M.; Xu, X.; Song, M.; Tao, H.; Bai, Y. Green tea epigallocatechin-3-gallate (EGCG) promotes neural progenitor cell proliferation and sonic hedgehog pathway activation during adult hippocampal neurogenesis. Mol. Nutr. Food Res. 2012, 56, 1292-1303. [CrossRef]

19. Yamashiro, T.; Tummers, M.; Thesleff, I. Expression of bone morphogenetic proteins and Msx genes during root formation. J. Dent. Res. 2003, 82, 172-176. [CrossRef] [PubMed]

20. Wang, W.; Dang, M.; Zhang, Z.; Hu, J.; Eyster, T.W.; Ni, L.; Ma, P.X. Dentin regeneration by stem cells of apical papilla on injectable nanofibrous microspheres and stimulated by controlled BMP-2 release. Acta Biomater. 2016, 36, 63-72. [CrossRef]

21. Qin, W.; Yang, F.; Deng, R.; Li, D.; Song, Z.; Tian, Y.; Wang, R.; Ling, J.; Lin, Z. Smad 1/5 is involved in bone morphogenetic protein-2-induced odontoblastic differentiation in human dental pulp cells. J. Endod. 2012, 38, 66-71. [CrossRef] [PubMed]

22. Feng, J.; Jing, J.; Li, J.; Zhao, H.; Punj, V.; Zhang, T.; Xu, J.; Chai, Y. BMP signaling orchestrates a transcriptional network to control the fate of mesenchymal stem cells in mice. Development 2017, 144, 2560-2569. [CrossRef]

23. Malik, Z.; Alexiou, M.; Hallgrimsson, B.; Economides, A.N.; Luder, H.U.; Graf, D. Bone Morphogenetic Protein 2 Coordinates Early Tooth Mineralization. J. Dent. Res. 2018, 97, 835-843. [CrossRef]

24. Aguilar, P.; Lertchirakarn, V. Comparison of stem cell behaviors between indigenous high and low-CD24 percentage expressing cells of stem cells from apical papilla (SCAPs). Tissue Cell 2016, 48, 397-406. [CrossRef]

25. Gosau, M.; Götz, W.; Felthaus, O.; Ettl, T.; Jäger, A.; Morsczeck, C. Comparison of the differentiation potential of neural crest derived progenitor cells from apical papilla (dNC-PCs) and stem cells from exfoliated deciduous teeth (SHED) into mineralising cells. Arch. Oral Biol. 2013, 58, 699-706. [CrossRef] [PubMed]

26. Bakopoulou, A.; Leyhausen, G.; Volk, J.; Tsiftsoglou, A.; Garefis, P.; Koidis, P.; Geurtsen, W. Comparative analysis of in vitro osteo/odontogenic differentiation potential of human dental pulp stem cells (DPSCs) and stem cells from the apical papilla (SCAP). Arch. Oral Biol. 2011, 56, 709-721. [CrossRef] [PubMed]

27. Gan, R.Y.; Li, H.B.; Sui, Z.Q.; Corke, H. Absorption, metabolism, anti-cancer effect and molecular targets of epigallocatechin gallate (EGCG): An updated review. Crit. Rev. Food Sci. Nutr. 2018, 58, 924-941. [CrossRef]

28. Irimie, A.I.; Braicu, C.; Zanoaga, O.; Pileczki, V.; Gherman, C.; Berindan-Neagoe, I.; Campian, R.S. Epigallocatechin-3-gallate suppresses cell proliferation and promotes apoptosis and autophagy in oral cancer SSC-4 cells. Onco Targets Ther. 2015, 8, 461-470. [CrossRef]

29. Singh, B.N.; Shankar, S.; Srivastava, R.K. Green tea catechin, epigallocatechin-3-gallate (EGCG): Mechanisms, perspectives and clinical applications. Biochem. Pharmacol. 2011, 82, 1807-1821. [CrossRef]

30. Mah, Y.J.; Song, J.S.; Kim, S.O.; Lee, J.H.; Jeon, M.; Jung, U.W.; Moon, S.J.; Kim, J.H.; Choi, H.J. The effect of epigallocatechin-3gallate (EGCG) on human alveolar bone cells both in vitro and in vivo. Arch. Oral Biol. 2014, 59, 539-549. [CrossRef] [PubMed]

31. Vali, B.; Rao, L.G.; El-Sohemy, A. Epigallocatechin-3-gallate increases the formation of mineralized bone nodules by human osteoblast-like cells. J. Nutr. Biochem. 2007, 18, 341-347. [CrossRef] [PubMed]

32. Chu, C.; Liu, L.; Wang, Y.; Yang, R.; Hu, C.; Rung, S.; Man, Y.; Qu, Y. Evaluation of epigallocatechin-3-gallate (EGCG)-modified scaffold determines macrophage recruitment. Mater. Sci. Eng. C Mater. Biol. Appl. 2019, 100, 505-513. [CrossRef] [PubMed] 
33. Castillo-Ortega, M.M.; Montaño-Figueroa, A.G.; Rodríguez-Félix, D.E.; Prado-Villegas, G.; Pino-Ocaño, K.P.; Valencia-Córdova, M.J.; Quiroz-Castillo, J.M.; Herrera-Franco, P.J. Preparation by coaxial electrospinning and characterization of membranes releasing (-) epicatechin as scaffold for tissue engineering. Mater. Sci. Eng. C Mater. Biol. Appl. 2015, 46, 184-189. [CrossRef]

34. Chu, C.; Deng, J.; Xiang, L.; Wu, Y.; Wei, X.; Qu, Y.; Man, Y. Evaluation of epigallocatechin-3-gallate (EGCG) cross-linked collagen membranes and concerns on osteoblasts. Mater. Sci. Eng. C Mater. Biol. Appl. 2016, 67, 386-394. [CrossRef] [PubMed]

35. Yang, J.W.; Zhang, Y.F.; Wan, C.Y.; Sun, Z.Y.; Nie, S.; Jian, S.J.; Zhang, L.; Song, G.T.; Chen, Z. Autophagy in SDF-1alpha-mediated DPSC migration and pulp regeneration. Biomaterials 2015, 44, 11-23. [CrossRef]

36. Li, S.; Lin, C.; Zhang, J.; Tao, H.; Liu, H.; Yuan, G.; Chen, Z. Quaking promotes the odontoblastic differentiation of human dental pulp stem cells. J. Cell Physiol. 2018, 233, 7292-7304. [CrossRef] [PubMed] 